
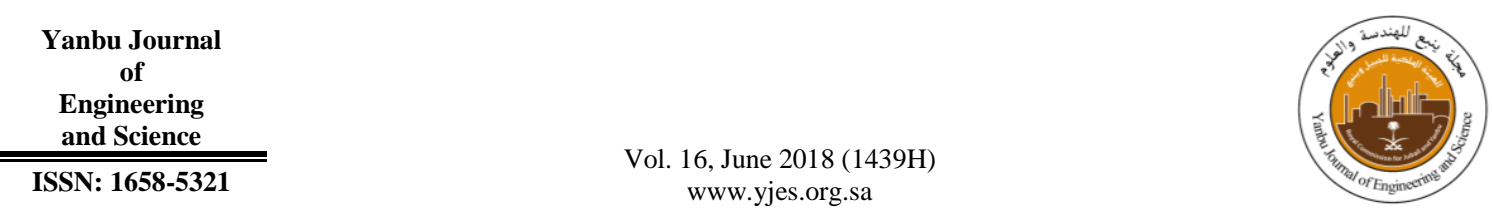

\title{
A THEORETICAL INVESTIGATION OF THE SCHRÖDINGER EQUATION FOR A MODIFIED TWO DIMENSIONAL PURELY SEXTIC DOUBLE-WELL POTENTIAL
}

\author{
Abdelmadjid Maireche \\ Laboratory of Physics and Material Chemistry, Physics department, Sciences Faculty, \\ University of M'sila-M'sila Algeria. \\ E-mail: abmaireche@gmail.com
}

\begin{abstract}
A theoretical analytical investigation for the exact solvability of non-relativistic quantum spectrum systems, at low energy for modified two-dimensional purely sextic double-well potential (2D-modified sextic DWAO potential) is discussed, by means Bopp's shift method, instead to solving deformed Schrödinger equation with star product, in the framework of both noncommutativite two dimensional real space and phase (NC: 2D-RSP), the exact corrections for lowest excitations are found straightforwardly for interactions in one-electron atoms by means of the standard perturbation theory for three special cases. Furthermore, the obtained corrections of energies are depended on the four infinitesimals parameters $(\theta, \chi)$ and $(\bar{\theta}, \bar{\sigma})$, which are induced by position-position and momentum-momentum noncommutativity, respectively, in addition to the discreet atomic quantum numbers $(j=l \pm 1 / 2, s= \pm 1 / 2, l)$ and the magnetic quantum number $m$. We have also generalized our obtained results to include the other atoms with $\operatorname{spin} \vec{S} \neq 1 / 2$.
\end{abstract}

Keywords: Two-dimensional purely sextic double-well potential, Noncommutative space and phase, Star product and Bopp's shift method

\section{INTRODUCTION}

The double-well anharmonic oscillator (DWAO) holds a great importance because of its relation to many problems of quantum chemistry and field theory [1-4]. Dai-Nam Le et al. was studied the Schrödinger equation for the purely sextic double-well potential problem in twodimensional space by wave function ansatz method for analytical approach and by Feranchuk-Komarov operator method for numerical approach [1]. Over the past few years, many efforts have been produced to study quantum systems on based to the Dirac equation, Klein-Gordon equation and Schrödinger equation, which is undoubtedly the most widely studied equation of modern physics, for spherical and non spherical potentials that in ordinary space of quantum mechanics and its extension, which known by extended quantum mechanics. The noncommutativity was introduced firstly by Heisenberg W. in 1930 [5] and then by Syndre H. in 1947 [6]. Recently, physicists have developed previously works by applying the noncommutativity properties on space and phase, the two ordinary generalized coordinates of space and momentums $\left(x^{\mu}, p^{\mu}\right)$ replaced by corresponding new values $\left(\hat{x}^{\mu}, \hat{p}^{\mu}\right)$, thus the two ordinary commutation $\left[x^{\mu}, x^{v}\right]$ 
and $\left[p^{\mu}, p^{v}\right]$ will be replace by two new commutation relations in NCQM $\left[\hat{x}^{\mu}, \hat{x}^{v}\right]$. and $\left[\hat{p}^{\mu}, \hat{p}^{v}\right]_{*}$ respectively (in natural units) [7-25]:

$\left[\hat{x}^{\mu}, \hat{x}^{v}\right]_{*}=i \theta^{\mu v}$ and $\left[\hat{p}^{\mu}, \hat{p}^{v}\right]_{*}=i \bar{\theta}^{\mu v}$

The symbol $(*)$ and the two parameters $\left(\theta^{\mu v}, \bar{\theta}^{\mu v}\right) \equiv \varepsilon^{\mu v}(\theta, \bar{\theta})$ are star product and antisymmetric real tensors induced by position-position and momentummomentum noncommutativity, respectively, it's important to notice that, the above two fundamental commutation relations are satisfied in particulars' cases from the general star product $f(x, p) * g(x, p)$ between two arbitrary functions $f(x, p)$ and $g(x, p)$ in the first order of two parameters $\theta^{\mu v}$ and $\bar{\theta}^{\mu v}$ as follow [18-32]:

$(f * g)(x, p)=(f g)(x, p)-\frac{i}{2} \theta^{\mu \nu} \frac{\partial f(x, p)}{\partial x^{\mu}} \frac{\partial g(x, p)}{\partial x^{v}}-\frac{i}{2}-\frac{\bar{\theta}}{\mu \nu} \frac{\partial f(x, p)}{\partial p^{\mu}} \frac{\partial g(x, p)}{\partial p^{\nu}}(2)$

The first term is the usual product in commutative space while the rest two parts are represents the effects of the noncommutativity of (space-space) and (phase-phase), respectively; a Bopp's shift method can be used, instead of solving any quantum systems by using directly star product procedure [28-34]:

$$
\left[\hat{x}_{i}, \hat{x}_{j}\right]=i \theta_{i j} \text { and }\left[\hat{p}_{i}, \hat{p}_{j}\right]=i \bar{\theta}_{i j}
$$

The generalized positions and momentum coordinates in the new 2D-NCQM $(\hat{x}, \hat{y})$ and $\left(\hat{p}_{x}, \hat{p}_{y}\right)$ generalized coordinates and momentums are depended with corresponding usual generalized positions and momentums coordinates $(x, y)$ and $\left(p_{x}, p_{y}\right)$, in the 2D-QM, by the following relations [27-36]:

$$
\begin{aligned}
& \hat{x}_{i}=x_{i}-\frac{\theta_{i j}}{2} p_{j} \Rightarrow \hat{x}=x-\frac{\theta}{2} p_{y} \text { and } \hat{y}=y+\frac{\theta}{2} p_{x} \\
& \hat{p}_{i}=p_{i}+\frac{\bar{\theta}_{i j}}{2} x_{j} \Rightarrow \hat{p}_{x}=p_{x}+\frac{\bar{\theta}}{2} y \text { and } \hat{p}_{y}=p_{x}-\frac{\bar{\theta}}{2} x
\end{aligned}
$$

Which allow us to getting the two operators $\hat{r}^{2}$ and $\hat{p}^{2}$ on a (NC: 2D-RSP) as follows [28-36, 38-42]:

$$
\left(r^{2}, p^{2}\right) \Rightarrow\left(\hat{r}^{2}, \hat{p}^{2}\right)=\left(r^{2}-\theta L_{z}, p^{2}+\bar{\theta} L_{z}\right)(5)
$$

With $L_{z} \equiv x p_{y}-y p_{x}$, this work is aimed at obtaining an analytic expression for the eigenenergies of a 2D-modified sextic DWAO potential in (NC: 2D-RSP) using the generalization Bopp's shift method based on mentioned formalisms on above equations to discover the new symmetries and a possibility to obtain another applications to this potential in different fields. The contents of the rest paper are as follows: In next section, we briefly review the Schrödinger equation with 2D-sextic DWAO potential. The Section 3, reserved to derive the 2D-modified sextic DWAO potential and perturbative terms of Hamiltonian operator using generalized Bopp's shift method for modified 2Dsextic DWAO potential. In the fourth section, we apply independent time standard perturbation theory to find the quantum spectrum of the lowest excitations in (NC: 2D RSP) for spinorbital interaction corresponding three special cases. In the next section, we deduce the new magnetic spectrum operator for studied potential. In the sixth section, we resume the global spectrum and corresponding noncommutative Hamiltonian for 2D-modified sextic DWAO potential. In the seventh section, we extended our study to include the energy spectra for other atoms with spin $\vec{S} \neq 1 \overrightarrow{/ 2}$ in (NC: 2D RSP). Finally, the important results and the conclusions are given in last section.

\section{REVIEW THE EIGNENFUNCTIONS AND THE ENERGY EIGENVALUES FOR 2D-SEXTIC DWAO POTENTIAL}

Let us present a brief review of time independent Schrödinger equation for a fermionic particle like electron of rest 
mass $M$ and its energy $E$ moving in 2Dsextic DWAO potential [1]:

$$
V(r)=-\frac{1}{2} M \omega^{2} \rho^{2}+\frac{\lambda}{8} \rho^{6}
$$

Here $\lambda$ is an anharmonicity constant and the parameter $\omega$ is real and positive. In the polar coordinates $(\rho, \varphi)$, the variables in the SE are separated and the wave functions can be presented in the form $\Psi(\rho, \varphi)=\frac{1}{\sqrt{2 \prod}} R(\rho) \exp (\operatorname{im} \varphi)$, with the magnetic quantum number ( $m=0, \pm 1, \pm 2, \ldots .$.$) , the radial wave$ function $R(\rho)$ is a solution of the following equation [1]:

$$
\left(\frac{d^{2}}{d \rho^{2}}+\frac{1}{\rho} \frac{d}{d \rho}-\frac{m^{2}}{\rho^{2}}+2 E+\beta \rho^{2}-\frac{1}{4} \rho^{6}\right) R(\rho)=0
$$

where $\beta=\frac{M^{3 / 2} \omega^{2}}{\hbar \lambda^{1 / 2}}$ is dimensionless and is referred to as a geometry parameter of the well. The wave function $\Psi_{E, m}(\rho, \varphi)$ in a general form [1]:

$$
\Psi_{E, m}(\rho, \varphi)=\frac{C_{0}}{\sqrt{2 \Pi}} \exp (i m \varphi) \rho^{\mu} \exp \left(-\frac{\rho^{4}}{8}\right) f(\rho)
$$

The function $f(\rho)$ is given by:

$$
f(\rho)=\sum_{k=0}^{\infty} C_{k} \rho^{2 k} \equiv \sum_{k=0}^{\infty} \frac{|m| !}{2^{\mathrm{k}} k !(\mathrm{k}+|m|) !} D_{k} \rho^{2 k}
$$

The coefficient $C_{0}$ is the normalization condition while $D_{k}=C_{2 k} \frac{2^{\mathrm{k}} k !(\mathrm{k}+|m|) !}{\mathrm{C}_{0}|m| !}$ with the coefficients $C_{2 k}$. The function $f(\rho)$ is able to become a polynomial of the value $\rho$, when the geometry values $\beta$ take a specific combined quantum number $(2 N+2+|m|)$, we are interested for study the following cases:

- The case of $N=0$ corresponds to the allowed geometry values $\beta=2+|m|$ (describing the quantum state with principal quantum number $n=1+|m|$ ) and the energy is equal to zero $E=0$, the wave function for this case is [1]:

$$
\Psi_{n=1+|m|, m}(\rho, \varphi)=\frac{1}{\sqrt{2 \Pi}} \rho^{|m|} \exp \left(-\frac{\rho^{4}}{8}\right) \exp (i m \varphi)(10)
$$

- For the case of $N=1$, the allowed geometry values are $\beta=4+|m|$, there are two solutions for energy $E_{n, m}$ as follows [1]:

$$
\begin{aligned}
& \Psi_{n=1+|m| m \mid m}(\rho, \varphi)=\frac{1}{\sqrt{2 \Pi}} \rho^{|m|} \exp \left(-\frac{\rho^{4}}{8}\right)\left(1+\frac{\rho^{2}}{\sqrt{4|m|+2}}\right) \exp (i m \varphi) \Rightarrow E_{n, m}=-\sqrt{2|m|+2} \\
& \Psi_{n=2+|m|, m}(\rho, \varphi)=\frac{1}{\sqrt{2 \Pi}} \rho^{|m|} \exp \left(-\frac{\rho^{4}}{8}\right)\left(1-\frac{\rho^{2}}{\sqrt{4|m|+2}}\right) \exp (i m \varphi) \Rightarrow E_{n, m}=\sqrt{2|m|+2}
\end{aligned}
$$

When $N>1$, the geometry values $\beta$ allowed more two values of principal quantum number, more two values of energy and more two values of waves functions (For more details please return to the ref. [1])

\section{THEORY OF (NC-2D: RSP) FOR 2D-MODIFIED SEXTIC DWAO POTENTIAL}

In this section, we shall study the 2Dmodified sextic DWAO potential in (NC: 2D-RSP), to perform this task the physical form of NR-SE, we apply the following steps [22-32]:

1. Ordinary two dimensional Hamiltonian operators $\hat{H}_{d w a o}\left(p_{i}, x_{i}\right)$ will be replace by new 2D Hamiltonian operators $\hat{H}_{n c-d w a o}\left(\hat{p}_{i}, \hat{x}_{i}\right)$, 
2. Ordinary complex wave function $\Psi(\vec{r})$ will be replacing by new complex wave function $\hat{\Psi}(\vec{r})$,

3. Ordinary energy $E_{n, m}$ will be replaced by new values $E_{n c-d w a o}$.

And the last step corresponds to replace the ordinary old product by new star product $(*)$, which allow us to constructing the 2D-DSE in both (NC-2D: RSP) for 2Dmodified sextic DWAO potential:

$$
\hat{H}_{n c-d w a o}\left(\hat{p}_{i}, \hat{x}_{i}\right) * \hat{\Psi}(\vec{r})=E_{n c-d w a o} \hat{\Psi}(\vec{r})
$$

It's important to notice that, the two obtained results in eq. (5) conserved the symmetry between $\left(\hat{p}_{i}\right.$ and $\hat{x}_{i}$ ) and the rolls of $\theta$ and $\bar{\theta}$ are inversed $(\theta \approx-\bar{\theta})$ [36]. The only none null two commutations relations: $\left\{[\hat{x}, \hat{y}]\right.$ and $\left.\left[\hat{p}_{x}, \hat{p}_{y}\right]\right\}$ and the two uncertainties relations $\left\{\Delta x \Delta y\right.$ and $\left.\Delta p_{x} \Delta p_{y}\right\}$ are determined from the following projections relations:

$$
\begin{array}{ll}
([\hat{x}, \hat{y}], & \left.\left[\hat{p}_{x}, \hat{p}_{y}\right]\right)=i(\theta,-\bar{\theta}) \\
(\Delta x \Delta y, & \left.\Delta p_{x} \Delta p_{y}\right) \approx(\theta, \bar{\theta})
\end{array}
$$

here $(\theta, \bar{\theta}) \equiv\left(\theta^{12}, \bar{\theta}^{12}\right)$, as mentioned before, we apply the Bopp's shift method on the equation (12) to obtain the reduced SE for 2D-modified sextic DWAO potential (Without star product):

$$
H\left(\hat{p}_{i}, \hat{x}_{i}\right) \psi(\vec{r})=E_{n c-d w a O} \psi(\vec{r})
$$

Where the new Hamiltonian operator $H\left(\hat{p}_{i}, \hat{x}_{i}\right)$ can be expressed in three general varieties: (NC-2D: RSP), (NC: 2D-RS) and (NC-2D: RP). In recently work, we are interest with the first variety (NC-2D: RSP):
$H_{n c-d u a o}\left(\hat{p}_{i}, \hat{x}_{i}\right) \equiv H\left(\hat{p}_{x}=p_{x}+\frac{\bar{\theta}}{2} y, \hat{p}_{y}=p_{y}-\frac{\bar{\theta}}{2} x, \hat{x}=x-\frac{\theta}{2} p_{y}, \hat{y}=y+\frac{\theta}{2} p_{x}\right)$ for NC-2D:RSP

After straightforward calculations, we can obtain the three important terms, which will be use to determine the 2D-modified sextic DWAO potential in (NC: 2D- RSP):

$$
\begin{aligned}
& -\frac{1}{2} M \omega^{2} \rho^{2} \rightarrow-\frac{1}{2} M \omega^{2} \hat{\rho}^{2}=-\frac{1}{2} M \omega^{2} \rho^{2}+\frac{1}{2} M \omega^{2} \theta L_{z} \\
& \frac{\lambda}{8} \rho^{6} \rightarrow \frac{\lambda}{8} \hat{\rho}^{6}=\frac{\lambda}{8} \rho^{6}-\frac{\lambda}{4} \rho^{4} \theta L_{z} \\
& \frac{p^{2}}{2 M} \rightarrow \frac{\hat{p}^{2}}{2 M}=\frac{p^{2}}{2 M}+\frac{\bar{\theta}}{2 M} L_{z}
\end{aligned}
$$

From above relations, one can write the formed the operator $V_{d w a o}(\hat{\rho})$ for $2 \mathrm{D}$ modified sextic DWAO potential and the noncommutative kinetic term $\frac{\hat{p}^{2}}{2 M}$, respectively:

$$
\begin{aligned}
& V_{d w a o}(\hat{\rho})=-\frac{1}{2} M \omega^{2} \hat{\rho}^{2}+\frac{\lambda}{8} \hat{\rho}^{6} \\
& \frac{\hat{p}^{2}}{2 M}=-\frac{1}{2 M}\left(\frac{1}{\rho} \frac{\partial}{\partial \rho}\left(\rho \frac{\partial}{\partial \rho}\right)+\frac{1}{\rho^{2}} \frac{\partial^{2}}{\partial \varphi^{2}}\right)+\frac{\bar{\theta} L_{z}}{2 M}
\end{aligned}
$$

Which allow us to obtaining the global potential operator $H_{\text {nc-dwao }}(\hat{\rho})$ for $2 \mathrm{D}$ modified sextic DWAO potential in (NC: 2D-RSP) as:

$$
H_{\text {ncdura }}(\hat{\rho})=-\frac{1}{2 M}\left(\frac{1}{\rho} \frac{\partial}{\partial \rho}\left(\rho \frac{\partial}{\partial \rho}\right)+\frac{1}{\rho^{2}} \frac{\partial^{2}}{\partial \varphi^{2}}\right)-\frac{1}{2} M \omega^{2} \rho^{2}+\frac{\lambda}{8} \rho^{6}+\left(\theta\left(\frac{1}{2} M \omega^{2}-\frac{\lambda}{4} \rho^{4}\right)+\frac{\bar{\theta}}{2 M}\right) L_{z}
$$

It's clearly, that the two first terms are given the ordinary 2D-sextic DWAO potential, while the rest terms are proportional's with two infinitesimals parameters ( $\theta$ and $\bar{\theta}$ ) and then gives the terms of perturbations Hamiltonian operator $H_{\text {per-dwao }}(\rho)$ in (NC: 2D-RSP) as:

$$
H_{\text {per-dwao }}(\rho)=\left(\theta\left(\frac{1}{2} M \omega^{2}-\frac{\lambda}{4} \rho^{4}\right)+\frac{\bar{\theta}}{2 M}\right) L_{z}
$$

It should be noted that there are many methods used in the solution of the Schrödinger equation, which related to the 
non-perturbative description of quantum systems, but our case is in this research related to the framework of the perturbative quantum systems, for further reading you can go back to the book [37].

\section{THE EXACT SPIN-ORBITAL}

SPECTRUM FOR 2D-MODIFIED

SEXTIC DWAO POTENTIAL IN NC:2DRSP) FOR ONE-ELECTRON ATOMS.

Again, the perturbative term $H_{\text {per-dwao }}(r)$ can be rewritten to the equivalent physical form for 2D-modified sextic DWAO potential, we have make the same physics procedure in our previous works [38-45]:

$$
H_{\text {per-dwao }}(r)=\gamma\left(\theta\left(\frac{1}{2} M \omega^{2}-\frac{\lambda}{4} \rho^{4}\right)+\frac{\bar{\theta}}{2 M}\right) \vec{L} \vec{S}
$$

Here $\gamma$ is the fine structure constante. The above result is logical for two reasons: the first one $\vec{S}=1 / 2$ (in one-electron atoms) and the second $\left(L_{x}=L_{y}=0\right)$, furthermore, the above perturbative term $H_{\text {per-dwao }}(\rho)$ can be rewritten to the following new equivalent form for 2D-modified sextic DWAO potential:

$$
H_{\text {per-dwao }}(\rho)=\gamma\left(\theta\left(\frac{1}{2} M \omega^{2}-\frac{\lambda}{4} \rho^{4}\right)+\frac{\bar{\theta}}{2 M}\right) G^{2}
$$

with $G^{2} \equiv \vec{J}^{2}-\vec{L}^{2}-\vec{S}^{2}$. To the best of our knowledge, we just replace the coupling spin-orbital $\vec{L} \vec{S}$ by the expression $\frac{1}{2}\left(\vec{J}^{2}-\vec{L}^{2}-\vec{S}^{2}\right)$, in quantum mechanics. The set $\left(H_{\text {so-dwao }}(\rho), \mathrm{J}^{2}\right.$, $\mathrm{L}^{2}, \mathrm{~S}^{2}$ and $J_{z}$ ) forms a complete of conserved physics quantities and the eigenvalues of the spin orbital coupling operator are

$k_{ \pm}(j=l \pm 1 / 2, l, s=1 / 2) \equiv \frac{1}{2}\left\{\left(l \pm \frac{1}{2}\right)\left(l \pm \frac{1}{2}+1\right)+l(l+1)-\frac{3}{4}\right\}$ corresponding $\quad j=l+\frac{1}{2} \quad$ (spin up) and $j=l-\frac{1}{2}$ (spin down), respectively, which allows us to form a diagonal $(2 \times 2)$ matrix, with non-null elements are $\left(H_{\text {dиаo }}^{\text {so }}\right)_{11}$ and $\left(H_{d \text { маo }}^{\text {so }}\right)_{22}$ for 2D-modified sextic DWAO potential in (NC: 2D-RSP) as:

$$
\begin{aligned}
& \left(H_{d a m o s}^{s o}\right)_{11}=k_{+} \gamma\left(\theta\left(\frac{1}{2} M \omega^{2}-\frac{\lambda}{4} \rho^{4}\right)+\frac{\bar{\theta}}{2 M}\right) \text { if } j=l+\frac{1}{2} \Rightarrow \text { spin - up } \\
& \left(H_{s, m o s}^{s o}\right)_{22}=k_{-} \gamma\left(\theta\left(\frac{1}{2} M \omega^{2}-\frac{\lambda}{4} \rho^{4}\right)+\frac{\bar{\theta}}{2 M}\right) \text { if } j=l-\frac{1}{2} \Rightarrow \text { spin - down }
\end{aligned}
$$

the variables in the DSE, which presented in eq. (14), are separated and the wave functions can be presented in the form $\Psi(\rho, \varphi)=\frac{1}{\sqrt{2 \Pi}} R(\rho) \exp (\operatorname{im} \varphi)$, the new radial wave function $R(\rho)$ is a solution of the following equation:

$\left(\frac{d^{2}}{d \rho^{2}}+E_{n c-d \text { mao }}+\frac{1}{2} M \omega^{2} \rho^{2}-\frac{\lambda}{8} \rho^{6}-\gamma\left(\theta\left(\frac{1}{2} M \omega^{2}-\frac{\lambda}{4} \rho^{4}\right)+\frac{\bar{\theta}}{2 M}\right) \vec{L} \vec{S}-\frac{m^{2}-\frac{1}{4}}{\rho^{2}}\right) R(r)=0$

It is clearly that the above equation including equation (20), the perturbative terms of Hamiltonian operator, which we are subject of discussion in next subsection, we look for a possibility to getting an exact solution of equation (23) including the added new part $H_{\text {per-dwao }}(r)$.

4.1 THE EXACT SPIN-ORBITAL SPECTRUM FOR 2D-MODIFIED SEXTIC DWAO POTENTIAL IN (NC: 2D- RSP) FOR ONE-ELECTRON ATOMS

In this subsection, we are going to study the modifications to the energy levels $\left(E_{\mathrm{u}-d w a o}^{(N)}\right.$ and $E_{\mathrm{u}-d w a o}^{(N)}$ ) for spin up and spin down, respectively, at first order of two parameters ( $\theta$ and $\bar{\theta}$ ) obtained by applying the standard perturbation theory, because the added new part $H_{\text {per-dwao }}(r)$, is very small compared to the principal part of 2D-modified sextic DWAO potential, which presented in eq. (6). We are interested to treat the following three principle cases: 
4.1.1 STUdY THE CASE $N=0$ AND GEOMETRY

$$
\begin{aligned}
& \text { VALUES } \beta=2+|m| \underset{\text { WITH PRINCIPAL }}{\text { WITH }} n=1+|m| \\
& \text { QUANTUM NUMBER }
\end{aligned}
$$

By applying the two axioms related to the energy

$$
\begin{aligned}
& E_{\mathrm{u}-d w a o}^{(1)}=\left\langle\Psi_{n=1+|m|, m}(\rho, \varphi)\left(H_{d \text { mao }}^{s o}\right)_{11} \Psi_{n=1+|m|, m}(\rho, \varphi)\right\rangle \\
& E_{\mathrm{d}-d w a o}^{(1)}=\left\langle\Psi_{n=1+|m|, m}(\rho, \varphi)\left(H_{d \text { wwo }}^{s o}\right)_{22} \Psi_{n=1+|+|, \mid, m}(\rho, \varphi)\right\rangle
\end{aligned}
$$

and using the wave equation in eq. (11), we may obtain the modifications to the energy levels for 2D-modified sextic DWAO potential $\left(E_{\mathrm{u}-d w a o}^{(0)}, E_{\mathrm{d}-d w a o}^{(0)}\right)$ for the case $\quad N=1$ and geometry values $\beta=4+|m|$ with principal quantum number $n=1+|m|$ :

$$
\begin{aligned}
& E_{\mathrm{u}-\mathrm{d} \text { wao }}^{(0)}=\left|N_{0}\right|^{2} \gamma k_{+}(j=l+1 / 2, l, s=1 / 2) \int_{0}^{+\infty} \rho^{2|m|+\mid+1} \exp \left(-\frac{\rho^{4}}{4}\right)\left(\theta\left(\frac{1}{2} M \omega^{2}-\frac{\lambda}{4} \rho^{4}\right)+\frac{\bar{\theta}}{2 M}\right) d \rho \\
& E_{\mathrm{d}-\mathrm{d} \text { wao }}^{(0)}=\left|N_{0}\right|^{2} \gamma k_{-}(j=l+1 / 2, l, s=1 / 2) \int_{0}^{+\infty} \rho^{2|m|+\mid+1} \exp \left(-\frac{\rho^{4}}{4}\right)\left(\theta\left(\frac{1}{2} M \omega^{2}-\frac{\lambda}{4} \rho^{4}\right)+\frac{\bar{\theta}}{2 M}\right) d \rho
\end{aligned}
$$

Another form to the above equations:

$$
\begin{aligned}
& E_{\mathrm{u}-d w a o}^{(0)}=\left|N_{0}\right|^{2} \gamma k_{+}(j=l+1 / 2, l, s=1 / 2)\left\{\theta \sum_{\mu=1}^{2} I_{0-\mu}^{s}+\frac{\bar{\theta}}{2 M} I_{0-3}^{p}\right\} \\
& E_{\mathrm{d}-d w a o}^{(0)}=\left|N_{0}\right|^{2} \gamma k_{-}(j=l+1 / 2, l, s=1 / 2)\left\{\theta \sum_{\mu=1}^{2} I_{0-\mu}^{s}+\frac{\bar{\theta}}{2 M} I_{0-3}^{p}\right\}
\end{aligned}
$$

Where, the three terms $I_{0-\mu}^{s}(i=\overline{1,2})$ and $I_{0-3}^{p}$ are given by:

$I_{0-1}^{s}=\frac{1}{2} M \omega^{2} I_{0-3}^{p}=\frac{1}{2} M \omega^{2} \int_{0}^{+\infty} \rho^{2|m|+1} \exp \left(-\frac{\rho^{4}}{4}\right) d \rho$,

$I_{0-2}^{s}=-\frac{\lambda}{4} \int_{0}^{+\infty} \rho^{2|m|+5} \exp \left(-\frac{\rho^{4}}{4}\right) d \rho$

It is convenient to using the following special integral [46]:

$$
\int_{0}^{+\infty} x^{\nu-1} \exp \left(-\mu x^{p}\right) d x=\frac{1}{p} \mu^{-\frac{v}{p}} \Gamma\left(\frac{v}{p}\right)
$$

With conditions $(\operatorname{Re} \mu>0, \operatorname{Re} v>0$ and $p>0)$ and $\Gamma\left(\frac{v}{p}\right)$ the ordinary Gamma function. After straightforward calculations, we can obtain the explicitly results:

$$
\begin{aligned}
& I_{0-1}^{s}=\frac{1}{2} M \omega^{2} I_{0-3}^{p}=\frac{2 M \omega^{2}}{4^{8|m|+8}} \Gamma(8|m|+8) \\
& I_{0-2}^{s}=-\frac{\lambda}{4^{8|m|+24}} \Gamma(8|m|+24)
\end{aligned}
$$

Which allow us to obtaining the exact modifications $\left(E_{\mathrm{u}-d w a o}^{(0)}\right.$ and $\left.E_{\mathrm{d}-d w a o}^{(0)}\right)$ produced by spin-orbital effect:

$$
\begin{aligned}
& E_{\mathrm{u}-d w a o}^{(0)}=\left|N_{0}\right|^{2} \gamma k_{+}(j=l+1 / 2, l, s=1 / 2)\left\{\theta I_{0-d w a o}^{s}+\frac{\bar{\theta}}{2 M} I_{0-d w a o}^{p}\right\} \\
& E_{\mathrm{d}-d w a o}^{(0)}=\left|N_{0}\right|^{2} \gamma k_{-}(j=l+1 / 2, l, s=1 / 2)\left\{\theta I_{0-d w a o}^{s}+\frac{\bar{\theta}}{2 M} I_{0-d w a o}^{p}\right\}
\end{aligned}
$$

we have introduced new coefficients $I_{0-d w a o}^{s}$ and $I_{0-d w a o}^{p}$ for the sake of simplicity:

$I_{0-d w a o}^{s} \equiv \frac{1}{4^{8|m|+8}}\left(2 M \omega^{2} \Gamma(8|m|+8)-\frac{\lambda}{4^{16}} \Gamma(8|m|+24)\right)$ and $I_{0-d \text { wao }}^{p}=\frac{4}{4^{8 \mid m+7}} \Gamma(8|m|+8)$

\subsubsection{STUdy THE CASE $N=1$ AND GEOMETRY VALUES $\beta=4+|m|$ WITH PRINCIPAL QUANTUM NUMBER $n=1+|m|$}

By applying the two axioms related to the energy

$$
E_{\mathrm{u}-d w a o}^{(1)}=\left\langle\Psi_{n=1+|m|, m}(\rho, \varphi)\left(H_{d \text { wao }}^{s o}\right)_{11} \Psi_{n=1+|m|, m}(\rho, \varphi)\right\rangle
$$

$E_{\mathrm{d}-d w a o}^{(1)}=\left\langle\Psi_{n=1+|m|, m}(\rho, \varphi)\left(H_{\text {dwao }}^{s o}\right)_{22} \Psi_{n=1+|m|, m}(\rho, \varphi)\right\rangle$ and using the wave equation in eq. (11), we may obtain the modifications to the energy levels for 2D-modified sextic DWAO potential $\left(E_{\mathrm{u}-d w a o}^{(0)}, E_{\mathrm{d}-d w a o}^{(0)}\right)$ for the case $\quad N=1$ and geometry values $\beta=4+|m|$ with principal quantum number $n=1+|m|$ :

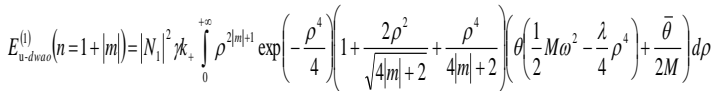

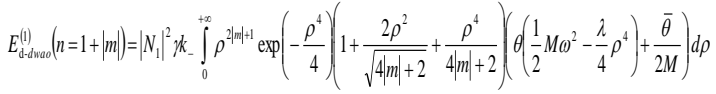

This can be rewritten as: 


$$
\begin{aligned}
& E_{\text {u.duaa }}^{(1)}(n=1+|m|)=\left|N_{1}\right|^{2} \gamma_{+}(j=l+1 / 2, l, s=1 / 2)\left\{\theta \sum_{\mu=1}^{6} I_{1-\mu}^{s}+\frac{\bar{\theta}}{2 M} \sum_{\nu=7}^{9} I_{1-\nu}^{p}\right\} \\
& E_{\text {d.dwao }}^{(1)}(n=1+|m|)=\left|N_{1}\right|^{2}{ }^{2} \mathcal{K}_{-}(j=l+1 / 2, l, s=1 / 2)\left\{\theta \sum_{\mu=1}^{6} I_{1-\mu}^{s}+\frac{\bar{\theta}}{2 M} \sum_{v=7}^{9} I_{1-\nu}^{p}\right\}
\end{aligned}
$$

Where, the 9-terms $I_{1-\mu}^{s}(\mu=\overline{1,6})$ and $I_{1-v}^{p}(v=\overline{7,9})$ are given by:

$$
\begin{aligned}
& I_{1-1}^{s}=\frac{1}{2} M \omega^{2} \int_{0}^{+\infty} \rho^{2|m|+2-1} \exp \left(-\frac{\rho^{4}}{4}\right) d \rho, \\
& I_{1-2}^{s}=\frac{M \omega^{2}}{\sqrt{4|m|+2}} \int_{0}^{+\infty} \rho^{2|m|+4-1} \exp \left(-\frac{\rho^{4}}{4}\right) d \rho, \\
& I_{1-3}^{s}=\frac{M \omega^{2}}{8|m|+4} \int_{0}^{+\infty} \rho^{2|m|+6-1} \exp \left(-\frac{\rho^{4}}{4}\right) d \rho \\
& I_{1-4}^{s}=-\frac{\lambda}{4} \int_{0}^{+\infty} \rho^{2|m|+6-1} \exp \left(-\frac{\rho^{4}}{4}\right) d \rho, \\
& I_{1-5}^{s}=-\frac{2 \lambda}{\sqrt{8|m|+4}} \int_{0}^{+\infty} \rho^{2|m|+8-1} \exp \left(-\frac{\rho^{4}}{4}\right) d \rho, \\
& I_{1-6}^{s}=-\frac{\lambda}{16|m|+8} \int_{0}^{+\infty} \rho^{2|m|+10-1} \exp \left(-\frac{\rho^{4}}{4}\right) d \rho \\
& I_{1-7}^{p}=\int_{0}^{+\infty} \rho^{2|m|+2-1} \exp \left(-\frac{\rho^{4}}{4}\right) d \rho, \\
& I_{1-8}^{p}=\frac{2}{\sqrt{4|m|+2}} \int_{0}^{+\infty} \rho^{2|m|+4-1} \exp \left(-\frac{\rho^{4}}{4}\right) d \rho, \\
& I_{1-9}^{p}=\frac{1}{4|m|+2} \int_{0}^{+\infty} \rho^{2|m|+6-1} \exp \left(-\frac{\rho^{4}}{4}\right) d \rho
\end{aligned}
$$

It's convenient to using the previously special integral, we can obtain the explicitly results:

$$
\begin{aligned}
& I_{1-1}^{s}=\frac{1}{8} M \omega^{2} 4^{\frac{|m|+1}{2}} \Gamma\left(\frac{|m|+1}{2}\right), \\
& I_{1-2}^{s}=\frac{M \omega^{2}}{4 \sqrt{4|m|+2}} 4^{\frac{|m|+2}{2}} \Gamma\left(\frac{|m|+2}{2}\right) \\
& I_{1-3}^{s}=\frac{M \omega^{2}}{32|m|+16} 4^{\frac{|m|+3}{2}} \Gamma\left(\frac{|m|+3}{2}\right)
\end{aligned}
$$

$$
\begin{aligned}
& I_{1-4}^{s}=-\frac{\lambda}{16} 4^{\frac{|m|+3}{2}} \Gamma\left(\frac{|m|+3}{2}\right), \\
& I_{1-5}^{s}=-\frac{2 \lambda}{4 \sqrt{8|m|+4}} 4^{\frac{|m|+4}{2}} \Gamma\left(\frac{|m|+4}{2}\right), \\
& I_{1-6}^{s}=-\frac{\lambda}{64|m|+32} 4^{\frac{|m|+5}{2}} \Gamma\left(\frac{|m|+5}{2}\right) \\
& I_{1-7}^{p}=\frac{1}{4} 4^{\frac{|m|+2}{2}} \Gamma\left(\frac{|m|+2}{2}\right), \\
& I_{1-8}^{p}=\frac{1}{\sqrt{16|m|+8}} 4^{\frac{|m|+2}{2}} \Gamma\left(\frac{|m|+2}{2}\right) \\
& I_{1-9}^{p}=\frac{1}{16|m|+8} 4^{\frac{|m|+3}{2}} \Gamma\left(\frac{|m|+3}{2}\right)
\end{aligned}
$$

Which allow us to obtaining the exact modifications

$\left(E_{\mathrm{u}-d w a o}^{(1)}(n=1+|m|)\right.$ and $\left.E_{\mathrm{d}-d w a o}^{(1)}(n=1+|m|)\right)$ produced by spin-orbital effect:

$$
\begin{aligned}
& E_{\text {d-dwao }}^{(1)}(n=1+|m|)=\left|N_{1}\right|^{2} \gamma k_{+}(j=l+1 / 2, l, s=1 / 2)\left\{\theta I_{1-\text { dwao }}^{s}+\frac{\bar{\theta}}{2 M} I_{1-\text { dwa }}^{p}\right\} \\
& E_{\text {d-dwao }}^{(1)}(n=1+|m|)=\left|N_{0}\right|^{2} \gamma_{-}(j=l+1 / 2, l, s=1 / 2)\left\{\theta I_{1-\text { dwao }}^{s}+\frac{\bar{\theta}}{2 M} I_{1-\text { dwao }}^{p}\right\}
\end{aligned}
$$

we have introduced new coefficients $I_{1-d w a o}^{s}$ and $I_{1-d w a o}^{p}$ for the sake of simplicity:

$$
I_{1-d w a o}^{s} \equiv \sum_{\mu=1}^{6} I_{1-\mu}^{s} \text { and } I_{1-d w a o}^{p}=\frac{\bar{\theta}}{2 M} \sum_{v=7}^{9} I_{1-v}^{p}
$$

4.1.3 STUdY THE CASE $N=1$ AND GEOMETRY VALUES $\beta=4+|m|$ WITH PRINCIPAL QUANTUM NUMBER $n=2+|m|$

By applying:

$$
E_{\mathrm{u}-d w a o}^{(1)}=\left\langle\Psi_{n=1+|m|, m}(\rho, \varphi)\left(H_{d w a o}^{s o}\right)_{11} \mid \Psi_{n=1+|| m \mid, m}(\rho, \varphi)\right\rangle
$$

and

$$
E_{\mathrm{d}-d w a o}^{(1)}=\left\langle\Psi_{n=1+|m|, m}(\rho, \varphi)\left(H_{\text {dwao }}^{s o}\right)_{22} \mid \Psi_{n=1+|m|, m}(\rho, \varphi)\right\rangle
$$

using the wave equation in eq. (10), we may obtain the modifications to the energy levels for 2D-modified sextic DWAO 
potential $\left(E_{\mathrm{u}-d w a o}^{(0)}, E_{\mathrm{d}-d w a o}^{(0)}\right)$ for the case $N=1$ and geometry values $\beta=4+|m|$ with principal quantum number $n=2+|m|$ :

$E_{u-d \text { wa }}^{(1)}(n=2+|m|)=\left|N_{1}\right|^{2} \psi_{+} \int_{0}^{+\infty} \rho^{2|m|+1} \exp \left(-\frac{\rho^{4}}{4}\right)\left(1-\frac{2 \rho^{2}}{\sqrt{4|m|+2}}+\frac{\rho^{4}}{4|m|+2}\right)\left(\theta\left(\frac{1}{2} M \omega^{2}-\frac{\lambda}{4} \rho^{4}\right)+\frac{\bar{\theta}}{2 M}\right) d \rho$ $E_{\mathrm{d}-d \mathrm{w} a \mathrm{o}}^{(\mathrm{l})}(n=2+|m|)=\left|N_{1}\right|^{2} \gamma k_{-} \int_{0}^{+\infty} \rho^{2|m|+1} \exp \left(-\frac{\rho^{4}}{4}\right)\left(1-\frac{2 \rho^{2}}{\sqrt{4|m|+2}}+\frac{\rho^{4}}{4|m|+2}\right)\left(\theta\left(\frac{1}{2} M \omega^{2}-\frac{\lambda}{4} \rho^{4}\right)+\frac{\bar{\theta}}{2 M}\right) d \rho$

An equivalent new form:

$E_{\mathrm{u}-d w a o}^{(1)}(n=2+|m|)=\left|N_{1}\right|^{2} \gamma k_{+}(j=l+1 / 2, l, s=1 / 2)\left\{\theta \sum_{\mu=1}^{6} T_{1-\mu}^{s}+\frac{\bar{\theta}}{2 M} \sum_{v=7}^{9} T_{1-\nu}^{p}\right\}$
$E_{\mathrm{d}-\text { dwao }}^{(1)}(n=2+|m|)=\left|N_{1}\right|^{2} \gamma k_{-}(j=l+1 / 2, l, s=1 / 2)\left\{\theta \sum_{\mu=1}^{6} T_{1-\mu}^{s}+\frac{\bar{\theta}}{2 M} \sum_{v=7}^{9} T_{1-\nu}^{p}\right\}$

Where, the 9-terms $T_{1-\mu}^{s}(\mu=1,6$
$T_{1-v}^{p}(v=7,9) \quad$ are $\quad$ given
$T_{1-1}^{s}=\frac{1}{2} M \omega^{2} \int_{0}^{+\infty} \rho^{2|m|+2-1} \exp \left(-\frac{\rho^{4}}{4}\right) d \rho$,

$T_{1-2}^{s}=-\frac{M \omega^{2}}{\sqrt{4|m|+2}} \int_{0}^{+\infty} \rho^{2|m|+4-1} \exp \left(-\frac{\rho^{4}}{4}\right) d \rho$

$T_{1-3}^{s}=\frac{M \omega^{2}}{8|m|+4} \int_{0}^{+\infty} \rho^{2|m|+6-1} \exp \left(-\frac{\rho^{4}}{4}\right) d \rho$

$T_{1-4}^{s}=-\frac{\lambda}{4} \int_{0}^{+\infty} \rho^{2|m|+6-1} \exp \left(-\frac{\rho^{4}}{4}\right) d \rho$

$T_{1-5}^{s}=\frac{2 \lambda}{\sqrt{8|m|+4}} \int_{0}^{+\infty} \rho^{2|m|+8-1} \exp \left(-\frac{\rho^{4}}{4}\right) d \rho$,

$T_{1-6}^{s}=-\frac{\lambda}{16|m|+8} \int_{0}^{+\infty} \rho^{2|m|+10-1} \exp \left(-\frac{\rho^{4}}{4}\right) d \rho$

$T_{1-7}^{p}=\int_{0}^{+\infty} \rho^{2|m|+2-1} \exp \left(-\frac{\rho^{4}}{4}\right) d \rho$

$T_{1-8}^{p}=-\frac{2}{\sqrt{4|m|+2}} \int_{0}^{+\infty} \rho^{2|m|+4-1} \exp \left(-\frac{\rho^{4}}{4}\right) d \rho$

$T_{1-9}^{p}=\frac{1}{4|m|+2} \int_{0}^{+\infty} \rho^{2|m|+6-1} \exp \left(-\frac{\rho^{4}}{4}\right) d \rho$

It is convenient to using the previously special integral, we can obtain the explicitly results:

$$
\begin{aligned}
T_{1-1}^{s} & =\frac{1}{8} M \omega^{2} 4^{\frac{|m|+1}{2}} \Gamma\left(\frac{|m|+1}{2}\right), \\
T_{1-2}^{s} & =-\frac{M \omega^{2}}{4 \sqrt{4|m|+2}} 4^{\frac{|m|+2}{2}} \Gamma\left(\frac{|m|+2}{2}\right) \\
T_{1-3}^{s} & =\frac{M \omega^{2}}{32|m|+16^{\frac{|m|+3}{2}}} \Gamma\left(\frac{|m|+3}{2}\right) \\
T_{1-4}^{s} & =-\frac{\lambda}{16} 4^{\frac{|m|+3}{2}} \Gamma\left(\frac{|m|+3}{2}\right), \\
T_{1-5}^{s} & =\frac{2 \lambda}{4 \sqrt{8|m|+4}} 4^{\frac{|m|+4}{2}} \Gamma\left(\frac{|m|+4}{2}\right), \\
T_{1-6}^{s} & =-\frac{\lambda}{64|m|+32} 4^{\frac{|m|+5}{2}} \Gamma\left(\frac{|m|+5}{2}\right) \\
T_{1-7}^{p} & =\frac{1}{4} 4^{\frac{|m|+2}{2}} \Gamma\left(\frac{|m|+2}{2}\right), \\
T_{1-8}^{p} & =-\frac{1}{\sqrt{16|m|+8}} 4^{\frac{|m|+2}{2}} \Gamma\left(\frac{|m|+2}{2}\right), \\
T_{1-9}^{p} & =\frac{1}{16|m|+8} 4^{\frac{|m|+3}{2}} \Gamma\left(\frac{|m|+3}{2}\right)
\end{aligned}
$$

Which allow us to obtaining the exact modifications $\left(E_{\mathrm{u}-d w a o}^{(1)}(n=2+|m|)\right.$ and $\left.E_{\mathrm{d}-d w a o}^{(1)}(n=2+|m|)\right)$ produced by spinorbital effect:

$E_{\mathrm{u}-d w a o}^{(1)}(n=2+|m|)=\left|N_{1}\right|^{2} \gamma k_{+}(j=l+1 / 2, l, s=1 / 2)\left\{\theta T_{1-d w a o}^{s}+\frac{\bar{\theta}}{2 M} T_{1-d w a o}^{p}\right\}$
$E_{\mathrm{d}-d \text { wao }}^{(1)}(n=2+|m|)=\left|N_{1}\right|^{2} \gamma k_{-}(j=l+1 / 2, l, s=1 / 2)\left\{\theta T_{1-d w a o}^{s}+\frac{\bar{\theta}}{2 M} T_{1-d w a o}^{p}\right\}$

we have introduced new coefficients $T_{1-d w a o}^{s}$ and $T_{1-d w a o}^{p}$ for the sake of simplicity:

$T_{1-d w a o}^{s} \equiv \sum_{\mu=1}^{6} T_{1-\mu}^{s}$ and $T_{1-d w a o}^{p}=\frac{\bar{\theta}}{2 M} \sum_{v=7}^{9} T_{1-\nu}^{p}$ 
5. THE EXACT MAGNETIC SPECTRUM FOR 2D-MODIFIED SEXTIC DWAO POTENTIAL IN (NC:2D- RSP)

On the other hand, we consider interested second physically meaningful phenomena, which produced automatically from the perturbative terms of 2D-modified sextic DWAO potential related to the influence of an external uniform magnetic field, it is sufficient to apply the following replacements to describing these phenomena:

$\left\{\begin{array}{l}\theta \rightarrow \chi B \\ \bar{\theta} \rightarrow \bar{\sigma}_{B}\end{array} \Rightarrow\left(\theta\left(\frac{1}{2} M \omega^{2}-\frac{\lambda}{4} \rho^{4}\right)+\frac{\bar{\theta}}{2 M}\right) L_{z} \rightarrow\left(\chi\left(\frac{1}{2} M \omega^{2}-\frac{\lambda}{4} \rho^{4}\right)+\frac{\bar{\sigma}}{2 M}\right) \vec{B} \vec{L}\right.$

here $\chi$ and $\bar{\sigma}$ are infinitesimal real proportional's constants, and we choose the magnetic field $\vec{B}=\mathbf{B} \vec{k}$, which allow us to introduce the modified new magnetic Hamiltonian $H_{m}^{d w a o}$ in (NC: 2D-RSP) as:

$$
H_{m}^{d w a o}=\left\{\chi\left(\frac{1}{2} M \omega^{2}-\frac{\lambda}{4} \rho^{4}\right)+\frac{\bar{\sigma}}{2 M}\right\}(\vec{B} \vec{J}-\vec{B} \vec{S})
$$

where $\vec{B} \vec{S}$ denote to the ordinary Hamiltonian of Zeeman Effect. To obtain the exact $\mathrm{NC}$ magnetic modifications of energy $\left(E_{\mathrm{m}-d w a o}^{(N)}(n)\right)$ for 2D-modified sextic DWAO potential corresponding the case $N=0$ and geometry values $\beta=2+|m|$ with principal quantum number $n=1+|m|$, the case $N=1$ and geometry values $\beta=4+|m|$ with principal quantum number $n=1+|m|$ and the case $N=1$ and geometry values $\beta=4+|m|$ with principal quantum number $n=2+|m|$, respectively, we make the three translations:

$k_{+} \rightarrow \mathrm{m}, \theta \rightarrow \chi$ and $\bar{\theta} \rightarrow \bar{\sigma}$

we insert the values of the parameters given in eq. (53) into eqs. (29), (39) and (49) to obtain ( $E_{\mathrm{m}-d w a o}^{(0)}(n=1+|m|), \quad E_{\mathrm{m}-d w a o}^{(1)}(n=1+|m|)$ and $\left.E_{\mathrm{m}-d w a o}^{(1)}(n=2+|m|)\right)$ as follows:

$$
\begin{aligned}
& E_{\mathrm{m}-\text { dwao }}^{(0)}(n=1+|m|)=\left|N_{0}\right|^{2} \gamma\left\{\chi I_{0-d w a o}^{s}+\frac{\bar{\sigma}}{2 M} I_{0-d w a o}^{p}\right\} B m \\
& E_{\mathrm{m}-\text { dwao }}^{(1)}(n=1+|m|)=\left|N_{1}\right|^{2} \gamma\left\{\chi I_{1-\text { dwao }}^{s}+\frac{\bar{\sigma}}{2 M} I_{1-d w a o}^{p}\right\} B m \\
& E_{\mathrm{m}-\text { dwao }}^{(1)}(n=2+|m|)=\left|N_{1}\right|^{2} \gamma\left\{\chi T_{1-\text { dwao }}^{s}+\frac{\bar{\sigma}}{2 M} T_{1-\text { dwao }}^{p}\right\} B m
\end{aligned}
$$

we have $-l \leq m \leq+l$, which allow us to fixing $(2 l+1)$ values for the orbital angular momentum quantum numbers.

\section{RESULTS}

Let us now resume the eigenenergies of the DSE obtained in this paper, the total modified energies ( $E_{n c-u d w a o}^{(N)}(n)$ and $E_{n c-u d w a o}^{(N)}(n)$ of a particle fermionic with spin up and spin down are determined corresponding fundamental states and first excited states, respectively, for 2Dmodified sextic DWAO potential in (NC: 2D-RSP), on based to the eqs. (9), (10), (29), (29), (49), and (54), we obtain the four new values of global energies:

The case $N=0$ and geometry values $\beta=2+|m|$ with principal quantum number $n=1+|m|$

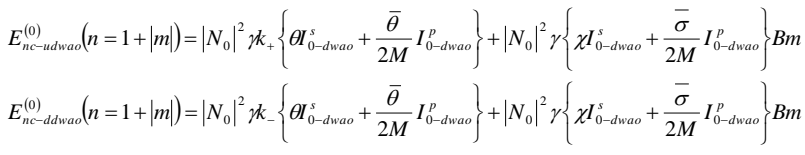

The case $N=1$ and geometry values $\beta=4+|m|$ with principal quantum number $n=1+|m|$ 


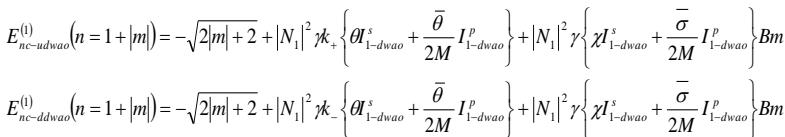

The case $N=1$ and geometry values $\beta=4+|m|$ with principal quantum number $n=2+|m|$ It's clearly, that the obtained eigenvalues of energies are real and then the NC diagonal Hamiltonian $H_{n c}^{d w a o}$ is Hermitian for 2D-modified sextic DWAO potential, furthermore it's possible to writing the non-null elements $\left(H_{n c}^{d w a o}\right)_{11}$ and $\left(H_{n c}^{d w a o}\right)_{22}$ as follows:

$$
\begin{aligned}
& \left(H_{n c}^{d w a o}\right)_{11}=-\frac{\Delta_{n c}}{2 M}+H_{\mathrm{int}}^{\text {dwao }} \\
& \left(H_{n c}^{d w a o}\right)_{22}=-\frac{\Delta_{n c}}{2 M}+H_{\mathrm{int}}^{d d w a o}
\end{aligned}
$$

Where the new kinetic energy is $\frac{\Delta_{n c}}{2 M}$ while the two modified interactions $H_{\text {int }}^{u d w a o}$ and $H_{\text {int }}^{d d w a o}$ for 2D-modified sextic DWAO potential are given by:

$$
\begin{aligned}
& \frac{\Delta_{n c}}{2 M}=\frac{\Delta-\bar{\theta} L_{z}-\bar{\sigma} L_{z}}{2 M} \\
& H_{\mathrm{int}}^{\text {udwao }}=-\frac{1}{2} M \omega^{2} \rho^{2}+\frac{\lambda}{8} \rho^{6}+\left(H_{d \text { миo }}^{s o}\right)_{11}+H_{m}^{d w a o} \\
& H_{\mathrm{int}}^{d d w a o}=-\frac{1}{2} M \omega^{2} \rho^{2}+\frac{\lambda}{8} \rho^{6}+\left(H_{d \text { миo }}^{s o}\right)_{22}+H_{m}^{d w a o}
\end{aligned}
$$

Thus, the ordinary kinetic term for 2DsexticDWAO potential $\left(-\frac{\Delta}{2 M}\right)$ and ordinary interaction $\left(-\frac{1}{2} M \omega^{2} \rho^{2}+\frac{\lambda}{8} \rho^{6}\right)$ are replaced by new modified form of kinetic term $\left(-\frac{\Delta_{n c}}{2 M}\right)$ and new modified

interactions ( $H_{\text {int }}^{u d w a o}$ and $H_{\text {int }}^{d d w a o}$ ). Which allows us to obtain the original results for this investigation: the obtained Hamiltonian operator (69) describing atom, which has two permanent dipoles: the first is electric dipole moment and the second is magnetic moment in external stationary electromagnetic field. Regarding to the wave functions for the case $N=2$ and geometry values $\beta=6+|m|$ with three principal quantum numbers $n=3+|m|$, $n=2+|m|$ and $n=1+|m|$, we can easily generalized our previously results to include the case $N=2$ and geometry values $\beta=6+|m|$ as follows: For the principal quantum number $n=3+|m|$ as follows:

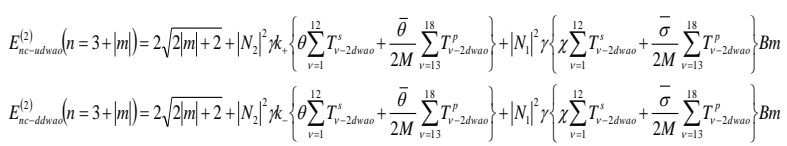

For the principal quantum number $n=2+|m|$, we have:

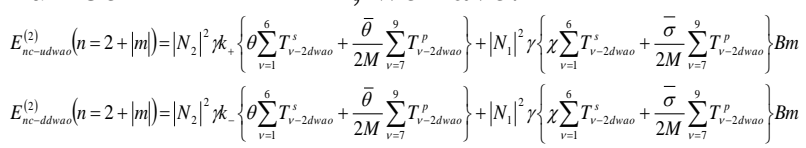

For the principal quantum number $n=1+|m|$ we have:

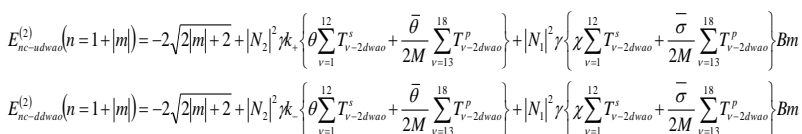

Now, we return to the degeneracy of energy level of 2D-modified sextic DWAO potential, we need to sum for all allowed values of $l$. Total degeneracy is thus, 
$2 \sum_{i=0}^{n-1}(2 l+1) \equiv 2 n^{2} \quad$ for the case $\mathrm{N}=0$ with one geometry values $2\left(2 \sum_{i=0}^{n-1}(2 l+1)\right) \equiv 4 n^{2}$ for the case $\mathrm{N}=1 \quad$ with two geometry values $3\left(2 \sum_{i=0}^{n-1}(2 l+1)\right) \equiv 6 n^{2}$ for the case $\mathrm{N}=2 \quad$ with threegeometry values

(62)

\section{GENERALIZATION THE OBTAINED} RESULTS TO THE ENERGY SPECTRA FOR THE ATOMS FOR $(\vec{S} \neq 1 \overrightarrow{1 / 2})$

As it is mentioned in the introduction, the 2D-modified sextic DWAO potential can be described the interactions in atoms with $\operatorname{spin} \vec{S} \neq 1 / 2$ in (NC: $2 \mathrm{D}-\mathrm{RSP}$ ), the atomic quantum number $m$ and $j$ can be takes

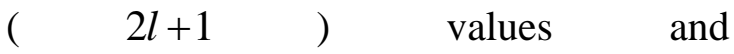
$\underbrace{j=|l-s|,|l-s|+1, \ldots, \ldots j=|l+s| \quad \text { and } \quad j=|l+s|}_{\text {N-possible valuesfor } \mathrm{j}}$ values, respectively, thus every old state in usually two dimensional space will be $N(2 l+1)$ sub-states for 2D-modified sextic DWAO potential. Furthermore, we can deduce the global noncommutative Hamiltonian $H_{n c-d w a o}$ in both (NC_2D: RSP) for studied potential on based to the previously obtained results:

$H_{n c-d \text { wao }}=-\frac{1}{2 M}\left(\frac{1}{\rho} \frac{\partial}{\partial \rho}\left(\rho \frac{\partial}{\partial \rho}\right)+\frac{1}{\rho^{2}} \frac{\partial^{2}}{\partial \varphi^{2}}\right)-\frac{1}{2} M \omega^{2} \rho^{2}+\frac{\lambda}{8} \rho^{6}+\gamma\left(\theta\left(\frac{1}{2} M \omega^{2}-\frac{\lambda}{4} \rho^{4}\right)+\frac{\bar{\theta}}{2 M}\right) \vec{L} \vec{S}+$ $+\left\{\chi\left(\frac{1}{2} M \omega^{2}-\frac{\lambda}{4} \rho^{4}\right)+\frac{\bar{\sigma}}{2 M}\right\}(\vec{B} \vec{J}-\vec{B} \vec{S})$

the corresponding energy spectra for three special cases: The case $N=0$ and geometry values $\beta=2+|m|$ with principal quantum number $n=1+|m|$ $E_{n c \text {-uwao }}^{(0)}(n=1+|m|)=\left|N_{0}\right|^{2} \gamma(j, l, s)\left\{\theta I_{0 \text {-dwaa }}^{s}+\frac{\bar{\theta}}{2 M} I_{0 \text {-dwao }}^{p}\right\}+\left|N_{0}\right|^{2} \gamma\left\{\chi I_{0 \text {-dwao }}^{s}+\frac{\bar{\sigma}}{2 M} I_{0 \text {-dwao }}^{p}\right\} B m$

The case $N=1$ and geometry values $\beta=4+|m|$ with principal quantum number $n=1+|m|$

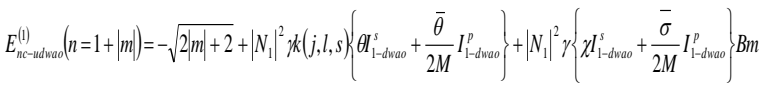

The case $N=1$ and geometry values $\beta=4+|m|$ with principal quantum number $n=2+|m|$

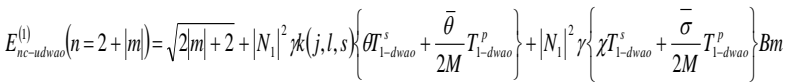

We have replaced the factor $k_{ \pm}(j=l \pm 1 / 2, l, s=1 / 2) \quad$ by new values $k(j, l, s) \equiv \frac{1}{2}\{j(j+1)+l(l+1)-s(s+1)\}$. Paying attention to the behaviour of the spectrums: (55), (56), (57), (59), (60), (61), (64), (65) and (66), it is possible to recover the results of commutative space when we consider $(\theta, \chi) \rightarrow(0,0)$.

\section{CONCLUSION}

To summarize, this work has been devoted to find the solutions of 2D-modified sextic DWAO potential, we have obtained the exact energy spectrum for three special cases: the case $N=0$ and geometry values $\beta=2+|m|$ with principal quantum number $n=1+|m|$, the case $N=1$ and geometry values $\beta=4+|m|$ with principal quantum number $n=1+|m|$ and the case $N=1$ and geometry values $\beta=4+|m|$ with principal quantum number $n=2+|m|$ in (NC: 2D-RSP) symmetries. We shown that the old states were changed radically and replaced by degenerated new states, describing two new original spectrums, the first one, produced by the of spin-orbital interaction while the second new spectrum produced by an external magnetic field, determined by the new results (55), (56), (57), (59), (60) and (61). Furthermore, our recently study can be generalized to 
including the interactions for atoms with $\operatorname{spin} \vec{S} \neq 1 / 2$ which describing by the new spectrums (64), (65) and (66).

\section{ACKNOWLEDGEMENT}

This work was supported with search laboratory of: Physics and Material Chemistry, in Physics department, Sciences faculty, University of M'sila, Algeria. The author would like to thank the kind's referees for the positive comments and suggestions, which led to the improved standard of this article.

\section{REFERENCES}

[1] Dai-Nam Le, Ngoc-Tram D. Hoang and VanHoang Le, "Exact analytical solutions of the Schrödinger equation for a two dimensional purely sextic double-well potential", Journal of Mathematical Physics 59, 2018, 032101 ; doi: $10.1063 / 1.4997532$

[2] Wen Z. C. and Yan Z. Dynamical, "behaviors of optical solitons in parity-time symmetric sextic anharmonic double-well potentials" Phys. Lett. A 379, 2015, 2025. DOI:https://doi.org/10.1016/j.physleta.2015.0 6.034

[3] Chaudhuri R. N. and Mondal M., "Eigenvalues of anharmonic oscillators and the perturbed Coulomb problem in Ndimensional space" Phys. Rev. A 52, 1995, 1850.

DOI:https://doi.org/10.1103/PhysRevA.52.18 50

[4] Hoang-Do N. T., Pham D. L. and Le V. H., Exact numerical solutions of the Schrödinger equation for a two-dimensional exciton in a constant magnetic field of arbitrary strength" Phys. B 423, 2013, 31. DOI: https://doi.org/10.1016/j.physb.2013.04.040.

[5] Heisenberg W., "Letter to R. Peierls (1930), in 'Wolfgang Pauli, Scienti- fic Correspondence', Vol. III, p.15, Ed. K. von Meyenn", (Springer Verlag 1985)

[6] Snyder H.,"The Quantization of space-time", Phys. Rev. 71 (1947) 38-41.

[7] Djemei A. E. F. and Smail H., "On Quantum Mechanics on Noncommutative Quantum
Phase Space" Commun. Theor. Phys. 41, 2004, 837-844.

[8] Joohan Lee, "Star Products and the Landau Problem. Journal of the Korean Physical Society" 47(4), 2005, 571-576.

[9] Jahan A., "Noncommutative harmonic oscillator at finite temperature: a path integral approach" Brazilian Journal of Physics, 37(4), 2007, 144-146.

[10] Yuan Y. e al., "Spin 1/2 relativistic particles in a magnetic field in NC Ph." Chinese Physics C, 34(5), 2010, 543.

[11] Jumakari-Mamat; Sayipjamal, "Dulat and Hekim Mamatabdulla, Landau-like Atomic Proplem on a Non-commutative Phase Space", Int J Theor Phys. (2016) DIO 10.1007/s10773-016-2922-1.

[12] Fateme Hoseini, Jayanta Saha K. and Hassan Hassanabadi, "Investigation of Fermions in Non-commutative Space by Considering Kratzer Potential", Commun. Theor. Phys. 65, 2016, 695-700.

[13] Castello-Branco K. H. C. and Martins A. G., "Free-fall in a uniform gravitational field in noncommutative quantum mechanics", JOURNAL OF MATHEMATICAL PHYSICS, 51, 2010, 102106.

[14] Bertolami O. and Leal P., "Aspects of phasespace noncommutative quantum mechanics, Physics Letters B. 2015, 750:6-11.

[15] Bastos C., Bertolami O., Dias N.C., Prata J.N., "Weyl-Wigner formulation of noncommutative quantum mechanics", J. Math. Phys. 49, 2008, 072101.

[16] Jian-zu Zhang, "Fractional angular momentum in non-commutative spaces", Physics Letters B 584, 2004, 204-209.

[17] Nair V. P. and Polychronakos A. P., "Quantum mechanics on the noncommutative plane and sphere", Physics Letters B. 505, 2001, , (1-4) 267-274.

[18] Gamboa J., Loewe M., and Rojas J. C. , "Noncommutative quantum mechanics", Phys. Rev. D 64, 2001, 067901. DOI:https://doi.org/10.1103/PhysRevD.64.06 7901.

[19] Scholtz F. G., Chakraborty B., Gangopadhyay S. and Hazra A. G., "Dual families of noncommutative quantum systems", Phys. Rev. D. 71, 2005, 085005 .

[20] Chaichian M., Sheikh-Jabbari M. M. and Tureanu A., "Hydrogen atom spectrum and the Lamb shift in noncommutative QED", Phys. Rev. Lett. 86, 2001, 2716. 
[21] Anirban Saha, Sunandan Gangopadhyay and Swarup Saha, "Quantum mechanical systems interacting with different polarizations of gravitational waves in noncommutative phase space”, PHYSICAL REVIEW D 97, 2018, 044015. DOI: 10.1103/PhysRevD.97.044015

[22] Abdelmadjid Maireche, "A Recent Study of Excited Energy Levels of Diatomics for Modified more General Exponential Screened Coulomb Potential: Extended Quantum Mechanics", J. Nano- Electron. Phys. 9(3), 201703021 DOI 10.21272/jnep.9(3).03021

[23] Abdelmadjid Maireche, "Investigations on the Relativistic Interactions in One-Electron Atoms with Modified Yukawa Potential for Spin 1/2 Particles", International Frontier Science Letters. 11, 2017, 29-44.

[24] Abdelmadjid Maireche, "New Relativistic Atomic Mass Spectra of Quark ( $u, d$ and s) for Extended Modified Cornell Potential in Nano and Plank's Scales", J. Nano- Electron. Phys. 8(1), 2016, 01020-1 - 01020-7.

[25] Abdelmadjid Maireche, "Investigations on the Relativistic Interactions in One-Electron Atoms with Modified Anharmonic Oscillator", J Nanomed Res 4(4), 2016, 00097. DOI: 10.15406/jnmr.2016.04.00097.

[26] Abdelmadjid Maireche, "New exact bound states solutions for (C.F.P.S.) potential in the case of Non-commutative three dimensional non relativistic quantum mechanics", Med. J. Model. Simul. 04, 2015, 060-072.

[27] Abdelmadjid Maireche, "A New Nonrelativistic Investigation for Interactions in One Electron Atoms with Modified Inverse-Square Potential: Noncommutative Two and Three Dimensional Space Phase Solutions at Planck's and Nano-Scales", J Nanomed Res 4(3), 2016, 00090. DOI: 10.15406/jnmr.2016.04.00090.

[28] Abdelmadjid Maireche, "New Bound State Energies for Spherical Quantum Dots in Presence of a Confining Potential Model at Nano and Plank's Scales", NanoWorld J, 1(4), 2016, 122-129.

[29] Abdelmadjid Maireche, "Quantum Hamiltonian and Spectrum of Schrödinger Equation with Companied Harmonic Oscillator Potential and its Inverse in Both Three Dimensional Non-commutative Real Space and Phase", J. Nano- Electron. Phys. 7 (4), 2015, 04021-1- 04021-7.

[30] Abdelmadjid Maireche, "A Complete Analytical Solution of the Mie-Type Potentials in Non-commutative 3-
Dimensional Spaces and Phases Symmetries", Afr. Rev Phys. 11, 2016, 111-117.

[31] Abdelmadjid Maireche, "A New Nonrelativistic Investigation for the Lowest Excitations States of Interactions in OneElectron Atoms, Muonic, Hadronic and Rydberg Atoms with Modified Inverse Power Potential", International Frontier Science Letters. 9, 2016, 33-46.

[32] Abdelmadjid Maireche, "New quantum atomic spectrum of Schrödinger equation with pseudo harmonic potential in both noncommutative three dimensional spaces and phases", Lat. Am. J. Phys. Educ. Vol. 9(1):1301 (2015).

[33] Abdelmadjid Maireche, "Deformed energy levels of a pseudoharmonic potential: nonrelativistic quantum mechanics", YJES. 12, 2016, 55-63.

[34] Abdelmadjid Maireche, "New Exact Solution of the Bound States for the Potential Family $\mathrm{V}(\mathrm{r})=\mathrm{A} / \mathrm{r} 2-\mathrm{B} / \mathrm{r}+\mathrm{Crk} \quad(\mathrm{k}=0,-1,-2)$ in both Noncommutative Three Dimensional Spaces and Phases: Non Relativistic Quantum Mechanics", International Letters of Chemistry, Physics and Astronomy. 58, 2015, 164-176.

[35] Abdelmadjid Maireche, "A New Approach to the Non Relativistic Schrödinger Equation for an Energy-Depended Potential $\mathrm{V}(\mathrm{r}, \mathrm{En}, 1)=\mathrm{V} 0(1+\eta \mathrm{En}, 1) \mathrm{r} 2$ in both Noncommutative Three Dimensional Spaces and Phases. International Letters of Chemistry", Physics and Astronomy.60: 11-19 (2015).

[36] Abdelmadjid Maireche, "New Bound States for Modified Vibrational-Rotational Structure of Supersingular Plus Coulomb Potential of the Schrödinger Equation in One-Electron Atoms", International Letters of Chemistry Physics and Astronomy. 73, 2017, 31-45.

[37] Feranchuk, I., Ivanov, A., Le, V.H., Ulyanenkov, A. "Non-perturbative Description of Quantum Systems", ISBN 9783-319-13006-4 Springer. Berlin-Heidelberg. 2015

[38] Abdelmadjid Maireche, "A New Theoretical Study of Quantum Atomic Energy Spectra for Lowest Excited States of Central (PIHOIQ) Potential in Noncommutative Spaces and Phases Symmetries at Plank's and Nanoscales", J. Nano- Electron. Phys. 8(1), 2016, 02027-1 - 02027-10.

[39] Abdelmadjid Maireche, "A New Nonrelativistic Atomic Energy Spectrum of 
Energy Dependent Potential for Heavy Quarkouniom in Noncommutative Spaces and Phases Symmetries", J. Nano- Electron. Phys. 8(2), 2016, 02046-1 - 02046-6.

[40] Abdelmadjid Maireche, "A new Nonrelativistic Investigation for Interactions in One-electron Atoms with Modified Vibrational-Rotational Analysis of Supersingular plus Quadratic Potential: Extended Quantum Mechanics", J. NanoElectron. Phys. 8(4), 2016, 04076-1- 04076-9.

[41] Abdelmadjid Maireche, "New Exact Energy Eigen-values for (MIQYH) and (MIQHM) Central Potentials: Non-relativistic Solutions", Afr. Rev Phys., 11, 2016, 175-184.

[42] Abdelmadjid Maireche, "The Exact Nonrelativistic Energy Eigenvalues for Modified Inversely Quadratic Yukawa Potential Plus Mie-type Potential", J. NanoElectron. Phys. 9(2), 2017, 1-7.
[43] Abdelmadjid Maireche, "Effects of TwoDimensional Noncommutative Theories on Bound States Schrödinger Diatomic Molecules under New Modified Kratzer-Type Interactions", International Letters of Chemistry", Physics and Astronomy 76, 2017, $1-11$.

[44] Abdelmadjid Maireche, "New Exact Nonrelativistic Energy Eigen Values for Modified Inversely Quadratic Hellmann Plus Inversely Quadratic Potential", J Nanosci Curr Res 2(3), 2017, 115. Doi: 10.4172/2572-0813.1000115.

[45] Abdelmadjid Maireche, "Effects of ThreeDimensional Noncommutative Theories on Bound States Schrödinger Molecular under New Modified Kratzer-type Interactions", J. Nano- Electron. Phys., 10(2), 02011-1 02011-6.

[46] Gradshteyn I. S. and Ryzhik I. M., Table of Integrals, Series and Products, 7th. ed.; Elsevier, edited by Alan Jeffrey (University of Newcastle upon Tyne, England) and Daniel Zwillinger (Rensselaer Polytechnic Institute USA) 2007. 
Yanbu Journal of Engineering and Science Vol. 16 (2018)

-55 - 
